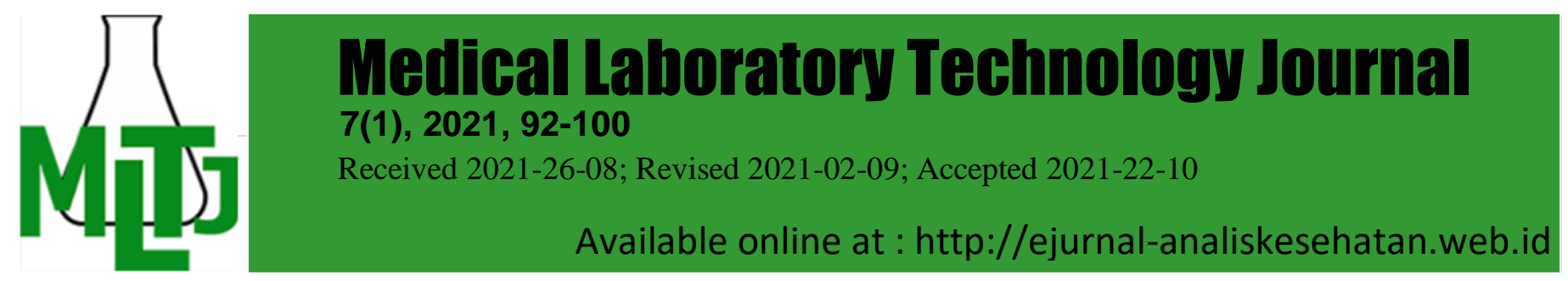

\title{
New Candidate Predisposition Genes for Hereditary Breast Cancer: SLIT3, CREB3, USP39
}

\author{
*Kirill Zagorodnev1, Alexandr Romanko1,2, Anna P. Sokolenko1,2, Ilya Bizin², \\ Ekaterina S. Kuligina ${ }^{2}$
}

${ }^{1}$ General and Medical genetics, Saint Petersburg State Pediatric Medical University, Saint Petersburg, Russia, 194100. '2Laboratory of Molecular Oncology, N.N. Petrov Institute of Oncology, Pesochny-2, St.-Petersburg, Russia, 197758

*Email: kirillzag93@gmail.com. DOI: 10.31964/mltj.v7i1.401

\begin{abstract}
Breast cancer is the most common type of malignant neoplasm in women. BRCA1 and BRCA2 are the most commonly mutated genes, but only up to $30 \%$ of hereditary breast cancer cases are attributed to alterations in these genes. A large proportion of genetic causes of hereditary breast cancer remains unknown. Thus, the search for new hereditary mutations and establishing a genetic alteration in each case of hereditary breast cancer is a clinically significant task; be the goal of our research. Next-generation sequencing (NGS) allows for simultaneous analysis of hundreds to thousands of genes at one time. We analyzed the genetic material of 49 patients of the northwest Russian population with clinical signs of hereditary breast cancer and identified new mutations associated with hereditary breast cancer. Research results show two missense mutations - SLIT3 p.Arg154Cys, CREB3 p.Lys157Glu, and truncating mutation - USP39 c. ${ }^{*} 208 \mathrm{G}>\mathrm{C}$. Research conclusion; The identified mutations can explain only a tiny fraction of hereditary breast cancer cases $(0.7 \%$ to $1.1 \%)$. The next step to increase the practical value of the detected alterations should be the analysis of biological characteristics of tumors in carriers of these mutations that can potentially become a target for chemotherapy.
\end{abstract}

Keywords: Hereditary breast and ovarian cancer; next generation sequencing; genetic oncology; breast cancer genetics; single-nucleotide polymorphism

\section{INTRODUCTION}

Breast cancer is the most common type of malignant neoplasm in women. About $5 \%$ to $10 \%$ of breast cancer cases are hereditary with genetic alterations in oncologically relevant regulator genes (Afghahi A. et al., 2017; Graffeo R. et al., 2016; Apostolou P. et al., 2013). Clinically significant signs of the hereditary nature of the disease are as follows: early-onset breast cancer ( $\leq 45$ years of age), bilateral tumors, family history of breast cancer, triple-negative breast cancer, male breast cancer (Afghahi A. et al., 2017; Couch FJ et al., 2017, Oldfield CJ et al., 2014). BRCA1 and BRCA2 are the most commonly mutated genes, but only up to $30 \%$ of hereditary breast cancer cases are attributed to alterations in these genes (Joseph L. et al., 2016; Lu HM et al., 2018). Another $10 \%-15 \%$ of hereditary breast cancer cases are associated with mutations in ATM, TP53, PALB2, PTEN, STK11, CHEK2, BARD1, BRIP1, CDH1, BLM, NF1, RAD51C (Kast K. et al., 2016; Hauke J. et al., 2018). A small fraction of cases (5\%-10\%) potentially can be caused by an unfavorable combination of several single nucleotide polymorphisms (SNPs) with low penetrance. There are dozens of such loci which are non-threatening individually but can be associated with increased cancer risk when combined. Therefore, a large proportion

Corresponding Author: Kirill Zagorodnev

General and Medical genetics, Saint Petersburg State Pediatric Medical University, Saint

Petersburg, Russia, 194100

Email: kirillzag93@gmail.com 
of genetic causes of hereditary breast cancer remains unknown. Meanwhile, understanding the molecular pathogenesis of hereditary cancer is crucial for developing personalized treatment and enhancing preventive measures (Nelson H.D. et al., 2014; $\mathrm{Ng} \mathrm{L.} \mathrm{et} \mathrm{al.,} \mathrm{2018).} \mathrm{Thus,} \mathrm{the} \mathrm{search} \mathrm{for} \mathrm{new} \mathrm{hereditary} \mathrm{mutations} \mathrm{and}$ establishing a genetic alteration in each case of hereditary breast cancer is a clinically significant task.

The breast cancer susceptibility genes, BRCA1 and BRCA2, were identified more than 20 years ago after extensive studies of families with several members with early-onset breast cancer. This approach was practical, but such studies can identify mostly high-penetrance genes, which limits the possibility of moderate and low penetrance genes discovery. New advances in genomic technologies have led to the development of next-generation sequencing (NGS), which has become a leading instrument in the search for new genetic alterations (Plon S. E. et al., 2018; Afghahi A. et al., 2017). This method allows to simultaneously analyze hundreds to thousands of genes at one time. Proper selection of patients plays a crucial role in NGS analysis. Typical criteria are early-onset breast cancer ( $\leq 45$ years of age) and bilateral tumors. It is important to note that genetic heterogeneity can significantly limit the study of hereditary breast cancer; conversely, the founder effect in some populations facilitates the research process.

Most of the known hereditary breast cancer mutations alter genome stability genes such as BRCA1/2and their partners ATM, TP53, PALB2, PTEN, STK11, CHEK2, BARD1, BRIP1, CDH1, BLM, NF1, RAD51C (Joseph L. et al., 2016; Kast K. et al., 2016; Hauke J. et al., 2018; Lu HM et al., 2018). Traditionally the search for causative mutation of breast cancer is limited to inactivating mutations in tumor suppressor genes. Still, the causes can likely be: a) alterations in genes not directly related to the DNA repair system, b) not only nonsense and frameshift mutations but also missense mutations associated with the loss of protein function.

NGS unveils many different genetic alterations and variants of uncertain significance (VUS) account for about $40 \%$ of total variants. These variants need to be accurately classified to determine their clinical significance and separate neutral from pathogenic variants (Federici G. et al., 2020). In 2004, Golghar and collaborators developed an algorithm that helps to determine the probability of each variant to be pathogenic (Goldgar DE et al., 2004). Criteria are as follows: frequency in case versus controls, co-occurrence with a known deleterious mutation, co-segregation with the disease in families, the occurrence of disease in relatives, and biochemical evidence such as residue position, conservation, and functional assays (Joseph L. et al., 2016). The proper assessment of discovered variant significance is essential. The International Agency for Research on Cancer (IARC) subdivides gene variants into five classes. Classes 1 and 2 include benign and likely benign variants, respectively. Classes 4 and 5 represent pathogenic and likely pathogenic variants, respectively. Class 3 includes VUS (Marlow R. et al., 2008; Federici G. et al., 2020). Class 3 is the most numerous, comprising about $40 \%$ of all variants discovered thus far (Balmana J. et al., 2016).

Most of the research devoted to the search for new genes associated with hereditary breast cancer focuses on inactivating mutations with apparent effects on the function of the genes. At the same time, the vast majority of coding genome variations are represented by missense mutations. Undoubtedly, many of them can contribute to the development of cancer. Currently, there are no effective methods for assessing the pathogenicity of amino acid substitutions. Identifying new mutations and assessing altered gene function has a pivotal role in hereditary breast cancer 
diagnosis and treatment. Thus, our study aims to identify novel mutations and evaluate the position of altered genes in the diagnosis and treatment of hereditary breast cancer.

\section{MATERIALS AND METHODS}

The Ethical Committee approved the study design of Saint Petersburg State Pediatric Medical University, Russia. All procedures performed in the study were by the 1964 Helsinki declaration and its later amendments or comparable ethical standards.

We analyzed the genetic material of 49 patients of the northwest Russian population with clinical signs of hereditary breast cancer. All patients included in the study did not have Slavic founder mutations in genes BRCA1, BRCA2, CHEK2, and NBS1. Additionally, we analyzed 18 healthy people who served as a control group to identify different variants occurring in the Russian population and exclude nonrelevant frequently encountered variants. WES was performed using Illumina NextSeq (33 samples) and Illumina MiSeq (16 samples) with the mean depth of coverage 36x for MiSeq and 81x for NextSeq. Nextera Rapid Capture Exome was used for library preparation and exome enrichment using Nextera Rapid Capture Exome kit (Illumina, USA).

Allele frequencies were studied using a case-control study in several cohorts of patients. The high-risk breast cancer group included 797 females (the mean age was 43); these patients received genetic testing in the Oncology institute named after $\mathrm{N}$. N. Petrov (Saint Petersburg, Russia) in 2008 - 2018 years and had one or more signs of predisposition to breast cancer (family history of breast cancer, bilateral tumors, early-onset breast cancer ( $<50$ years of age). We also studied 1505 consecutive patients (the mean age was 57) who had genetic testing in the Oncology institute named after N. N. Petrov (Saint Petersburg, Russia) in 2008 - 2018. The control group included 1070 healthy females (the mean age was 44).

Likely pathogenic "candidate" variants were genotyped using High-Resolution Melt Analysis (HRM) with subsequent Sanger sequencing (Figure 1) or by real-time allele-specific PCR (AS-PCR) using CFX96 Real-Time PCR System (Bio-Rad. the USA). Genotype frequencies in the patients and healthy controls groups were compared using chi-square and two-sided Fisher's exact tests.

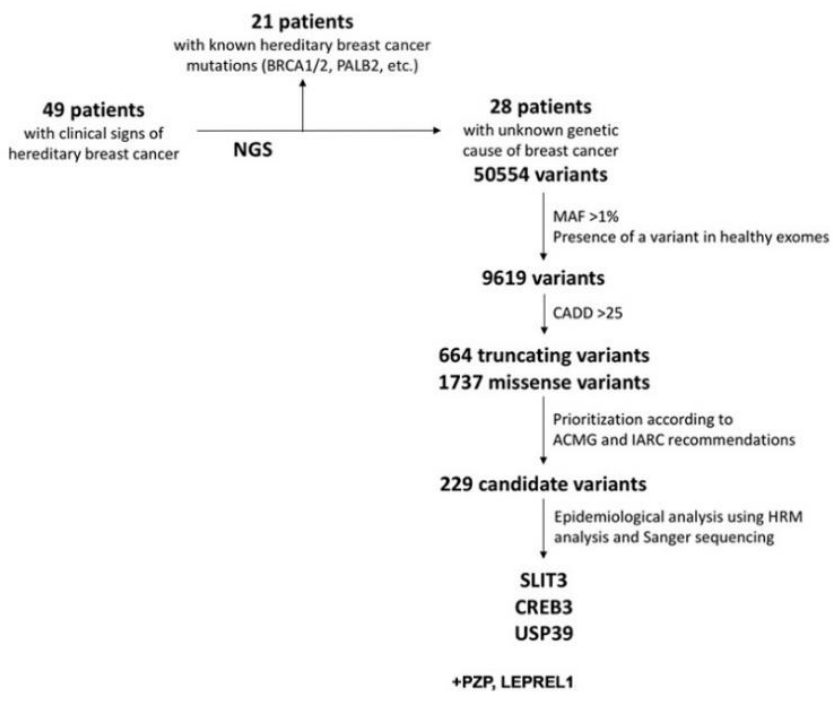

Figure 1. Algorithm of Candidate Mutation Selection 


\section{RESULTS AND DISCUSSION}

In 21/49 (42.9\%) patients, we found hereditary mutations in genes with a known oncological role: BRCA1, BRCA2, PALB2, BLM, RAD51C, RAD50, RAD54L, FANCM, WRN, MMS22L, and ERCC4. The analysis of the remaining 28 patients identified 50554 alternative variants. Still, a significant part of it was filtered due to high minor allele frequency (MAF) in the population (more than 1\% according to the ExAC database) or because of the presence of these variants in our collection of healthy exomes. In such a way, we reduced the list to 9,619 variants. Subsequently, we selected truncating mutations $(n=664)$ and missense mutations with a CADD-score $>25(n=1737)$. The remaining variants were prioritized according to recommendations of the American College of Medical Genetics (ACMG) and the International Agency for Research on Cancer (IARC), and preference was given to pathogenic and probably pathogenic variants (Goldgar DE et al., 2004).

We also considered the data of the variant occurrence in cancer patients and healthy individuals according to the gnomAD database (https://gnomad.broadinstitute.org/), a more than 2-fold increase of variant detection among cancer patients was considered as an argument in favor of its potential oncogenic role. It helped calculate the odds ratio (OR) of getting breast cancer being a carrier of these mutations. As a result, we made a list of 229 candidate mutations. The next step was the comparison of allele frequencies in groups of breast cancer patients and healthy women. For these purposes, we used High-Resolution Melt Analysis (HRM) with subsequent Sanger sequencing. Thus, the predisposing role was confirmed for three mutations: two missense mutations with high in silico pathogenicity - SLIT3 p.Arg154Cys (CADD = 31) and CREB3 p.Lys157Glu (CADD = 29.3) and truncating mutation - USP39 c. ${ }^{*} 208 \mathrm{G}>\mathrm{C}$ (Table 1).

Table 1. Frequency of Detected Mutations High-risk group Consecutive group Healthy women

\begin{tabular}{cccccccc}
\hline Gene & $\begin{array}{c}\text { Mutation } \\
\text { frequency }\end{array}$ & $\begin{array}{c}\text { OR(95\% } \\
\text { Cl) }\end{array}$ & $\begin{array}{c}\boldsymbol{P} \text { value } \\
\text { frequency }\end{array}$ & $\begin{array}{c}\text { Mutation } \\
\text { fr) }\end{array}$ & $\begin{array}{c}\boldsymbol{P} \\
\text { value }\end{array}$ & $\begin{array}{c}\text { Mutation } \\
\text { frequency }\end{array}$ \\
\hline SLIT3 & $9 / 834$ & 3.99 & .024 & $14 / 1239$ & 3.66 & .043 & $3 / 1024$ \\
& $(1.1 \%)$ & $(1.198-$ & & $(1.1 \%)$ & $(1.041-$ & & $(0.03 \%)$ \\
& & $13.270)$ & & & $12.886)$ & & \\
\hline CREB & $6 / 832$ & 7.34 & .06 & $3 / 1242$ & $2.45(0.254-$ & .439 & $1 / 1012$ \\
3 & $(0.72 \%)$ & $(0.882-$ & 5 & $(0.24 \%)$ & $23.570)$ & & $(0.098 \%)$ \\
& & $61.134)$ & & & & & \\
\hline USP39 & $6 / 792$ & 17.6 & .05 & $9 / 1340$ & $15.2(0.89-$ & .06 & $0 / 1066$ \\
& $(0.75 \%)$ & $(1.00-$ & & $(0.67 \%)$ & $261.79)$ & & \\
& & $312.60)$ & & & & & \\
\hline
\end{tabular}

The frequency of the SLIT3 p.Arg154Cys mutation was $1.1 \%$ in High-risk and Consecutive groups (9/834 and 14/1239, respectively) which was significantly higher than in the group of healthy women - 3/1024 (0.03\%). OR (high-risk), $3.99 ; 95 \% \mathrm{Cl}$, 1.198 - 13.270; $P=0.024$, OR (consecutive) $3.66 ; 95 \% \mathrm{Cl}, 1.041-12.886 ; \mathrm{P}=0.043$. The highest frequency of the CREB3 p.Lys157Glu mutation was recorded in High-risk patients $6 / 832(0.72 \%)$. In Consecutive group we found the mutation in $3 / 1242(0.24 \%)$ patients, and in the healthy women in $1 / 1012(0.098 \%)$. OR (high-risk), $7.34 ; 95 \% \mathrm{Cl}$, $0.882-61.134 ; \mathrm{P}=0.065$, and $\mathrm{OR}$ (consecutive), $2.45 ; 95 \% \mathrm{Cl} 0.254-23.570 ; \mathrm{P}=$ 0.439 . The frequency of mutation USP39 c. ${ }^{*} 208 \mathrm{G}>\mathrm{C}$ in the High-risk group was $6 / 792$ 
$(0.75 \%)$, and in Consecutive group - $9 / 1340(0.67 \%)$. It is noteworthy that in a group of healthy women $(1,066)$ the mutation was not found. OR (high-risk), $17.6 ; 95 \% \mathrm{Cl}$, 1.00 - 312.60; $\mathrm{P}=0.05$ ), OR (consecutive), 15.2; 95\% Cl, $0.89-261.79 ; \mathrm{P}=0.06$. We also found that the carriage of the USP39 c. ${ }^{*} 208 \mathrm{G}>\mathrm{C}$ mutation was associated with the development of triple-negative breast cancer $(P=0.0001)$, and the SLIT3 p.Arg154Cys mutation was associated with the multifocal breast cancer $(P=0.022)$ (Table 2).

Table 2. Clinical Signs of Hereditary Disease in Patients with Detected Mutations

\begin{tabular}{ccccccccc}
\hline \multirow{2}{*}{ Gene } & \multicolumn{2}{c}{ Family history } & \multicolumn{2}{c}{$\begin{array}{c}\text { Early-onset } \\
\text { breast cancer }\end{array}$} & \multicolumn{2}{c}{$\begin{array}{c}\text { Multifocal breast } \\
\text { cancer }\end{array}$} & \multicolumn{2}{c}{$\begin{array}{c}\text { Triple-negative } \\
\text { breast cancer }\end{array}$} \\
\cline { 2 - 9 } & Yes & No & Yes & No & Yes & No & Yes & No \\
\multirow{2}{*}{ SLIT3 } & $2 / 302$ & $21 / 1702$ & $12 / 950$ & $11 / 1054$ & $\mathbf{5} / \mathbf{1 4 5}$ & $18 / 1859$ & $5 / 223$ & $13 / 1004$ \\
& $(0.66 \%)$ & $(1.23 \%)$ & $(1.26 \%)$ & $(1.04 \%)$ & $(3.45 \%)$ & $(0.97 \%)$ & $(2.2 \%)$ & $(1.3 \%)$ \\
\multirow{2}{*}{ CREB3 } & $3 / 304$ & $5 / 1711$ & $5 / 958$ & $3 / 1057$ & $1 / 149$ & $7 / 1866$ & $1 / 223$ & $6 / 1010$ \\
& $(1.00 \%)$ & $(0.29 \%)$ & $(0.52 \%)$ & $(0.28 \%)$ & $(0.67 \%)$ & $(0.38 \%)$ & $(0.4 \%)$ & $(0.6 \%)$ \\
\multirow{2}{*}{ USP39 } & $2 / 314$ & $13 / 1818$ & $8 / 974$ & $7 / 1158$ & 0.158 & $15 / 1974$ & $\mathbf{1 1 / 2 6 4}$ & $3 / 1159$ \\
& $(0.64 \%)$ & $(0.72 \%)$ & $(0.82 \%)$ & $(0.60 \%)$ & & $(0.76 \%)$ & $(\mathbf{4 . 2} \%)$ & $(0.26 \%)$ \\
\hline
\end{tabular}

We also identified two more mutations that can contribute to the development of hereditary breast cancer but were encountered more rarely in tested patients: PZP p.Arg680*, LEPREL1 p.Pro636Ser (Table 3).

The frequency of PZP p.Arg680* mutation in High-risk group was 4/1046 (0.38\%) and in Consecutive group - $9 / 1608(0.56 \%)$. The mutation was once identified in the group of healthy women $-1 / 1194(0.0008 \%) .5 / 13$ patients with breast cancer and PZP mutation had multifocal carcinomas - bilateral breast cancer, breast cancer, and colorectal cancer, breast cancer and gastric carcinoma, breast cancer, and basal cell carcinoma. The LEPREL1 p.Pro636Ser mutation was identified in 4/1054 (0.37\%) in the High-risk group and 5/1500 (0.33\%) in the Consecutive group. The LEPREL1 mutation was also identified once in the group of healthy women 1/1067 (0.0009\%). $7 / 9$ patients with this mutation had estrogen-positive breast cancer.

Table 3. Frequency of PZP and LEPREL1 Mutations in Tested Patients High-risk group Consecutive group Healthy women

\begin{tabular}{cccccccc}
\hline Gene & $\begin{array}{c}\text { Mutation } \\
\text { frequency }\end{array}$ & $\begin{array}{c}\text { OR(95 } \\
\% \text { Cl) }\end{array}$ & $\begin{array}{c}\boldsymbol{P} \text { value } \\
\text { vutation } \\
\text { frequency }\end{array}$ & $\begin{array}{c}\text { OR(95 } \\
\% \text { Cl) }\end{array}$ & $\begin{array}{c}\boldsymbol{P} \\
\text { value }\end{array}$ & $\begin{array}{c}\text { Mutation } \\
\text { frequency }\end{array}$ \\
\hline$P Z P$ & $4 / 1046$ & 6.08 & .087 & $9 / 1608$ & 4.15 & .201 & $1 / 1194$ \\
& $(0.38 \%)$ & $(0.770-$ & & $(0.56 \%)$ & $(0.464-$ & & $(.0008 \%)$ \\
& & $48.096)$ & & & $37.224)$ & & \\
\hline LEPREL & $4 / 1054$ & 4.05 & .210 & $5 / 1500$ & 3.57 & .246 & $1 / 1067$ \\
1 & $(0.37 \%)$ & $(0.453-$ & & $(0.33 \%)$ & $(0.416-$ & & $(.0009 \%)$ \\
& & $36.395)$ & & & $30.561)$ & & \\
\hline
\end{tabular}

This study revealed a possible contribution of identified genes to hereditary breast cancer. Confirming their oncogenic role will explain only a minor fraction of hereditary breast cancer cases. However, such low-risk variants are still an excellent addition to testing panels because exploring new susceptibility genes improves individual cancer risk prediction. These newly identified mutations were not previously mentioned in association with hereditary breast cancer. However, their participation in 
regulating oncologically critical cellular processes has been repeatedly described in the literature.

The SLIT3 gene is widely expressed in mammalian tissues, and its deregulations have been identified in malignant tissues. The product of the SLIT3 gene -multiple EGF-like domains protein five is involved in regulating cell motility, angiogenesis, and proliferation. The SLIT3 gene was already mentioned earlier as a tumor suppressor gene in thyroid cancer, colorectal cancer, gastric cancer, nasopharyngeal carcinoma, cervical cancer, ovarian cancer, and pancreatic ductal adenocarcinoma (Zhang C. et al., 2015; Ng L. et al., 2018). Moreover, in a mouse model of breast cancer, stimulation of SLIT3 expression was associated with the suppression of tumor cells growth (Marlow R. et al., 2008) and impairment of cancer cell invasion and migration through modulation of the expressions of E-cadherin, Vimentin, MMP2, and MMP9 (Zhang C. et al., 2015).

The CREB3 gene is expressed in numerous cell types. Its product (cyclic AMPresponsive element-binding protein 3 ) is involved in regulating proliferation, cell migration, and stress-induced protein degradation (Zhao F et al., 2016; Howley BV et al., 2018). Alteration in this gene plays a significant role in cervical cancer progression via the c-Jun-MMP9 axis causing enhanced expression of MMP9, a biomarker for tumor metastasis (Park E. et al., 2014). It is also involved in prostate cancer development (Kim Y. et al., 2015). Moreover, CREB3 could enhance the expression of Bcl-2, an essential anti-apoptotic molecule in the plasma (Wu Y. et al., 2019).

The product of the USP39 gene (ubiquitin specific peptidase 39, or snRNP assembly defective one homolog) plays an essential role in pre-mRNA splicing and regulates cell division. Upregulation of USP39 is associated with the stimulation of tumor cell growth in vitro and in vivo (Yuan X. et al., 2015). Knockdown of USP39 gene inhibits cell proliferation in breast, stomach, and liver, and also induces apoptosis in tumor cells (Wang H. et al., 2013; Xing Z. et al., 2018; Xu Y. et al., 2018; Wang YA et al., 2019; Talhouet S. et al., 2020). Furthermore, the USP39 deubiquitinase has recently been identified as an upstream regulator of the checkpoint kinase 2 (CHEK2) a product of a known breast cancer susceptibility gene (Wu J. et al., 2019).

This study was limited by the lack of genetic segregation data because we could not analyze the genetic material of relatives of our patients. Despite the likely proteininactivating effect of discovered mutations and their potentially oncogenic properties, it is essential to conduct functional analysis in cell lines and segregation analysis in families with breast cancer. The final proof of the significance of identified mutations would be the structural and functional analysis of the altered proteins in vitro and various in vivo tests.

The application of Next Generation Sequencing has facilitated multigene panel analysis and became a leading instrument in the search for new genetic alterations. This approach helps to personalize the management of patients with hereditary breast cancer. Tsaousis G. et al., 2019 conducted similar research and were able to identify 264 pathogenic variants out of 1197 individuals with personal or family history of breast cancer $(22.1 \%)$, while a VUS was identified in $34.8 \%(417 / 1197)$ of the cases; the analysis of only BRCA1/2 in this cohort explained just $10.5 \%$ of genetic alterations, so this research helped them to expand the multigene panel used in practice.

\section{CONCLUSION}

This study suggests the role of these rare variants in predisposition to hereditary breast cancer. The identified mutations can explain only a tiny fraction of hereditary breast cancer cases $(0.7 \%$ to $1.1 \%)$. However, these genes may be a significant 
addition to the targeted cancer panels used for molecular diagnosis of hereditary breast cancer using next-generation sequencing (NGS). The next step to increase the practical value of the detected alterations should be the analysis of biological characteristics of tumors in carriers of these mutations that can potentially become a target for chemotherapy.

\section{ACKNOWLEDGMENTS}

The research was supported by Russian Foundation for Basic Research (Grant 19-315-90086)

\section{CONFLICT OF INTEREST}

The authors declare that they have no conflict of interest.

\section{REFERENCES}

Afghahi A., Kurian W. (2017). A the changing landscape of genetic testing for inherited breast cancer predisposition. Current treatment options in oncology, 18(27), 10.1007/s11864-017-0468-y

Apostolou P., Fostira F. (2013). Hereditary breast cancer: the era of new susceptibility genes. BioMed Research International, -P, 1-11. 10.1155/2013/747318

Balmana J., Digiovanni L, Gaddam P, et al. (2016). Conflicting interpretation of genetic variants and Cancer risk by commercial laboratories as assessed by the prospective registry of multiplex testing. Journal of Clinical Oncology, 34, 40714078. 10.1200/JCO.2016.68.4316

Couch FJ, Shimelis H, Hu C, et al. (2017). Associations between cancer predisposition testing panel genes and breast cancer. JAMA Oncology, 3, 11901196. 10.1001/jamaoncol.2017.0424

Federici, G., Soddu, S. (2020). Variants of uncertain significance in the era of highthroughput genome sequencing: a lesson from breast and ovary cancers. Journal of Experimental \& Clinical Cancer Research, 39, 10.1186/s13046-02001554-6

Goldgar DE, Easton DF, Deffenbaugh AM, et al. (2004). Integrated evaluation of DNA sequence variants of unknown clinical significance: application to BRCA1 and BRCA2. The American Journal of Human Genetics, 75, 535544. $10.1086 / 424388$

Graffeo R., Livraghi L. (2016). Time to incorporate germline multigene panel testing into breast and ovarian cancer patient care. Breast Cancer Res treat, 160, 393410. 10.1007/s10549-016-4003-9

Hauke J., Horvath J., Groß E. (2018). Gene panel testing of 5589 BRCA1/2-negative index patients with breast cancer in a routine diagnostic setting: results of the German Consortium for Hereditary Breast and ovarian Cancer. Cancer Med, 7 , 1349-1358. 10.1002/cam4.1376

Howley BV, Link LA, Grelet S, et al. (2018). A CREB3-regulated ER-Golgi trafficking signature promotes metastatic progression in breast cancer. Oncogene, 37, 1308-1325. 10.1038/s41388-017-0023-0

Joseph L, Cankovic M, Caughron S, et al. (2016). The Spectrum of clinical Utilities in Molecular Pathology Testing Procedures for inherited conditions and Cancer: a report of the Association for Molecular Pathology. The Journal of Molecular Diagnostics, 18, 605-619. 10.1016/j.jmoldx.2016.05.007 
Kast K., Rhiem K., Wappenschmidt B. (2016). Prevalence of BRCA1/2 germline mutations in 21401 families with breast and ovarian cancer. J. Med. Genet, 53, 465-471. 10.1136/jmedgenet-2015-103672

Kim Y, Kim J, Jang SW, Ko J. (2015). The role of SLZIP in cyclin D3-mediated negative regulation of androgen receptor transactivation and its involvement in prostate cancer. Oncogene, 34, 226-236. 10.1038/onc.2013.538

Lu H.M., Li s., Black M.H., Lee s. et al. (2018). Association of Breast and ovarian Cancers With Predisposition Genes Identified by Large-scale sequencing. JAMA oncol, 10.1001/jamaoncol.2018.2956

Marlow R, Strickland P, Lee JS, et al. (2008). SLITs suppress tumor growth in vivo by silencing Sdf1/Cxcr4 within breast epithelium. Cancer Research, 68, 78197827. 10.1158/0008-5472.CAN-08-1357

Nelson H.D., Pappas M., Zakher B. (2014). Risk assessment, genetic counseling, and genetic testing for BRCA- related cancer in women: a systematic review to update the U.S. Preventive services task Force recommendation. Ann Intern. Med, 160, 255-266. 10.7326/M13-1684

$\mathrm{Ng} \mathrm{L,} \mathrm{Chow} \mathrm{AKM,} \mathrm{Man} \mathrm{JHW,} \mathrm{et} \mathrm{al.} \mathrm{(2018).} \mathrm{Suppression} \mathrm{of} \mathrm{Slit3} \mathrm{induces} \mathrm{tumor}$ proliferation and chemoresistance in hepatocellular carcinoma through activation of GSK3 $\beta / \beta$-catenin pathway. BMC Cancer, 18, 1. 10.1186/s12885-018-4326-5

Oldfield CJ, Dunker AK. (2014). Intrinsically disordered proteins and intrinsically disordered protein regions. Annu Rev Biochem, 83, 553-584. $10.2174 / 092986610791498984$

Park E, Kang H, Kim J, Ko J. (2014). The role of sLZIP in transcriptional regulation of c-Jun and involvement in migration and invasion of cervical cancer cells. Cell Physiol Biochem, 33, 151-164. 10.1159/000356658

Plon, S. E., Eccles, D. M., Easton, D. (2008). Sequence variant classification and reporting: recommendations for improving the interpretation of cancer susceptibility genetic test results. Human Mutation, 29, 12821291. 10.1002/humu.20880

Talhouet S, Peron J, Vuilleumier A, et al. (2020). Clinical outcome of breast cancer in carriers of BRCA1 and BRCA2 mutations according to molecular subtypes. Scientific Reports, 10.1038/s41598-020-63759-1

Tsaousis, G.N., Papadopoulou, E., Apessos, A. et al. (2019). Analysis of hereditary cancer syndromes by using a panel of genes: novel and multiple pathogenic mutations. BMC Cancer, 19, 535. https://doi.org/10.1186/s12885-019-5756-4

Wang H, Ji X, Liu X, et al. (2013). Lentivirus-mediated inhibition of USP39 suppresses the growth of breast cancer cells in vitro. Oncology Reports, 30, 28712877. 10.3892/or.2013.2798

Wang YA, Jian JW, Hung CF, et al. (2018). Germline breast cancer susceptibility gene mutations and breast cancer outcomes. BMC Cancer, 18, 10.1186/s12885-0184229-5

Wu J, Chen Y, Geng G, Li L, Yin P, Nowsheen S, Li Y: Wu C, Liu J, Zhao F, Kim W, Zhou Q, Huang J, Guo G, Zhang C, Tu X, Gao X, Lou Z, Luo K, Qiao H, Yuan J. (2019). USP39 regulates DNA damage response and chemo-radiation resistance by deubiquitinating and stabilizing CHK2. Cancer Lett, 1, 114-124. 10.1016/j.canlet.2019.02.015.

Wu Y, Xie Z, Chen J. (2019). Circular RNA circTADA2A promotes osteosarcoma progression and metastasis by sponging miR-203a-3p and regulating CREB3 expression. Mol Cancer, 2, 73. 10.1186/s12943-019-1007-1. PMID: 30940151 PMCID: PMC6444890 
Xing Z, Sun F, He W, et al. (2018). Downregulation of ubiquitin-specific peptidase 39 suppresses the proliferation and induces the apoptosis of human colorectal cancer cells. Oncology Letters, 15, 5443-5450. 10.3892/ol.2018.8061

Xu Y, Zhu MR, Zhang JY, et al. (2018). Knockdown of ubiquitin-specific peptidase 39 inhibits the malignant progression of human renal cell carcinoma. Molecular Medicine Reports, 17, 4729-4735. 10.3892/mmr.2018.8421

Yuan X, Sun X, Shi X, et al. (2015). USP39 promotes the growth of human hepatocellular carcinoma in vitro and in vivo. Oncology Reports, 34, 823832. 10.3892/or.2015.4065

Zhang C, Guo H, Li B, et al. (2015). Effects of Slit3 silencing on the invasive ability of lung carcinoma A549 cells. Oncology Reports, 34, 952960. 10.3892/or.2015.4031

Zhao F, Wang N, Yi Y, et al. (2016). Knockdown of CREB3/Luman by shRNA in Mouse Granulosa Cells Results in Decreased Estradiol and Progesterone Synthesis and Promotes Cell Proliferation. PLOS ONE, 11, 10.1371/journal.pone.0168246. 